appealing study. These two flaws, if addressed, would improve the conclusions one could draw from future studies as well as defining a separate behavioural profile specific to the syndrome.

Denies, M. Udwin, O. \& Howlin, P. (1990) Adults with Williams syndrome. Preliminary study of social, emotional and behavioural difficulties. British fournol of Psychiotry, I72, 273-276.

P. S. S. Russell Child and Adolescent Psychiatry Unit, Christian Medical College, Vellore 632002, India

Author's reply: Full details of the adaptive behaviour and cognitive profile of this group are published elsewhere (see Udwin et al, 1996; Davies et al, 1997; Howlin et al, 1998). Briefly, it was found that differences between verbal and performance IQ, and between receptive and expressive language skills, were smaller than generally found in children with this condition, although the cognitive profile of abilities and difficulties remains almost identical to that found in children with Williams syndrome. Hyperacusis remains prominent in adulthood but it is not regarded as being as disruptive as in childhood.

With regard to recruiting participants, ensuring the generalisability of findings is a perennial problem for research of this nature. It is important that studies aim to recruit as many participants as possible. It is often very difficult to identify and recruit large numbers of people with such rare syndromes, and parent groups often represent the most efficient means of doing so. Such families are often extremely motivated to participate in research, as demonstrated by the extremely high response rate to our study. It is difficult to speculate whether the group of adults recruited in this study is truly representative of the population as a whole. However, it seems likely that families with no support or understanding of Williams syndrome will encounter more difficulties coping with the challenges presented by this condition. Moreover, given the high degree of behavioural disturbance and levels of care required by our group, it is likely that many undiagnosed adults with Williams syndrome are cared for by professional agencies. It is therefore important that those likely to encounter individuals such as those with Williams syndrome are aware of associated behavioural phenotypes.
Davies, M., Howlin, P. \& Udwin, Q (ISes) Independence and adaptive behaviour in adults with Williams syndrome. Americon foumal of Medical Genetics, 70. 188-195.

Howlin, P., Devies, M. \& Udwin, O. (1998) Cognitive functioning in adults with Williams syndrome. Joumal of Child Psychology and Psychiatry, 39, 183-189.

Udwin, O., Devies, M. \& Howlin, P. (ISA) A longitudinal study of cognitive ability and educational attainment in Williams syndrome. Developmental Medicine and Child Neurology. 38, 1020-1029.

M. Davies School of Psychology, David Keir Building, Queen's University of Belfast, Malone Road, Belfast BT7 INN

\section{White matter abnormalities and memory in Alzheimer's disease}

Sir: Ikeda et al (1998) draw attention to the heterogeneity of memory impairment even in advanced Alzheimer's disease and the authors discuss the possible role of the amygdala and its connections in emotional memory. Given that all the subjects had magnetic resonance imaging scans, and despite the relatively small sample size, it would be interesting to know whether any imaging findings were related to the preservation or not of memory for the emotionally charged event. In particular, since white matter abnormalities are frequently present in Alzheimer's disease (Kertesz et $a l, 1990)$ and since memory loss has been described in association with damage to white matter projections from the amygdala (Kooistra \& Heilman, 1988), it could be hypothesised that such abnormalities would be more frequent in the subjects who forgot the earthquake, for a given stage of dementia severity.

Ilboda, M., Mori, E., Hirono, N., et ol (1990) Amnestic people with Alzheimer's disease who remembered the Kobe earthquake. British fournal of Psychiatry 172. $425-428$.

Kertesz, A., Polk, M. \& Carr, T. (1990) Cognition and white matter changes on magnetic resonance imaging in dementia. Archives of Neurology, 47, 387-391.

Koolstra, C. A. \& Hellman, K. M. (1988) Memory loss from a subcortical white matter infarct. Journol of Neurology, Neurosurgery and Psychiatry, 51. 866-869.

\section{R. J. Stewart Institute of Psychiatry,}

De Crespigny Park, London SE5 8AF

Authors' reply: We thank Dr Stewart for his interest in our study and his legitimate comment on magnetic resonance imaging (MRI) findings related to the emotionally charged event. In several MRI-based volumetric studies, medial temporal atrophy correlates to memory impairment in $\mathrm{Alz}$ heimer's disease (Mori et al, 1997b). In fact, we quantified amygdalar and hippocampal volumes in a subset of our patients by using high-resolution MRI and a computerised volumetric technique to elucidate the relationship between medial temporal damage and memory for the events surrounding the earthquake. We found that, irrespective of generalised brain atrophy and cognitive impairments, amygdalar volume (right and left averaged) correlated with emotional memory more than hippocampal volume did. General knowledge of the earthquake was correlated with neither amygdalar nor hippocampal volume. The findings indicate that impairment of emotional event memory in patients with Alzheimer's disease is related to intensity of amygdalar damage, and provide evidence of the amygdala's involvement in emotional memory in humans. A part of this study was presented at the American Neurological Association annual meeting (Mori et al, 1997a).

As Dr Stewart pointed out, it is plausible that white matter abnormalities are involved in the memory impairment of the subjects of our study. In people with Alzheimer's disease, white matter abnormalities are often noted on T2-weighted magnetic resonance images, and are reportedly involved in cognitive impairment. However, we did not include in the study those with severe white matter abnormalities whose characteristics otherwise fulfilled the criteria for clinical diagnosis of Alzheimer's disease, because of a possible involvement of ischaemic pathology. Moreover, a recent study suggested that white matter abnormalities in people with Alzheimer's disease is not a disease-specific change but an age-associated coincidence that has little relevance to cognitive function and thus little clinical importance (Leys et al, 1990; Marder et al, 1995). In a previous study, we studied the impact of T2-weighted MRI white matter hyperintensities on cerebral perfusion and oxygen metabolism in patients with Alzheimer's disease by using oxygen-15 steady-state method and positron emission tomography (Yamaji et al, 1997). We found no significant difference in cognitive impairment between those with and without white matter abnormalities. There were no significant differences in oxygen metabolism in cortical and white matter regions 